\title{
sciendo
}

\author{
BULGARIAN ACADEMY OF SCIENCES
}

CYBERNETICS AND INFORMATION TECHNOLOGIES • Volume 21, No 2

Sofia $2021 \quad$ Print ISSN: 1311-9702; Online ISSN: 1314-4081

DOI: $10.2478 /$ cait-2021-0018

\section{Performance Evaluation of Change Detection in SAR Images Based on Hybrid Antlion DWT Fuzzy c-Means Clustering}

\author{
J. Thrisul Kumar ${ }^{1}$, B. M. S. Rani ${ }^{1}$, M. Satish Kumar ${ }^{2}$, M. V. Raju ${ }^{3}$, \\ K. Maria Das ${ }^{3}$ \\ ${ }^{1}$ ECE Dept, Vignan's Nirula Inst of Sci and Tech for Women, India \\ ${ }^{2}$ Kallam Haranadha Reddy Inst of Technology, India \\ ${ }^{3}$ Vignan's Foundation for Science Technology and Research, India \\ E-mails:_kumarthrisul9@gmail.com_ranibms@gmail.com_satish.envi@gmail.com \\ rajumv.environ@gmail.com mariadaskarri@gmail.com
}

\begin{abstract}
In this paper, the main objective is to detect changes in the geographical area of Ottawa city in Canada due to floods. Two multi-temporal Synthetic Aperture Radar (SAR) images have been taken to evaluate the un-supervised change detection process. In this process, two ratio operators named as Log-Ratio and Mean-Ratio are used to generate a difference image. Performing image fusion based on DWT by selecting optimum filter coefficients by satisfying the wavelet filter coefficient properties through a novel image fusion technique is named as ADWT. GA, PSO, AntLion Optimization algorithms (ALO) and Hybridized AntLion Algorithm (HALO) have been adapted to perform the ADWT based image fusion. Segmentation has been performed based on fuzzy c-Means clustering to detect changed and unchanged pixels. Finally, the performance of the proposed method will be analysed by comparing the segmented image with the ground truth image in terms of sensitivity, accuracy, specificity, precision, F1-score.
\end{abstract}

Keywords: ADWT, ALO Algorithm, HALO Algorithm, GA Algorithm, PSO Algorithm.

\section{Introduction}

SAR is able to generate images in all weather conditions that means it is capable of producing images in the night as well as in cloudy conditions. It can monitor the objects and is able to generate images 24 hours irrespective [1] of atmospheric conditions. Usually, the change detection process is performed in three steps. In the first step, image pre-processing will be performed, in the second step the difference image will be generated and finally, changes could be detected. It is a known fact that the segmentation can be done by using clustering, where clustering is the grouping of similarities, which indicates that separation between similar and non-similar 
objectives could be possible. Based on this concept [1] detection of changes can be performed by using image fusion and clustering. Change detection can be attained by using Adaptive Discrete Wavelet Transform (ADWT) with Fuzzy c-Means (FCM) clustering [2-5].

Image fusion has been employed [1] to extract more information and to achieve symmetry between the altered pixel and unaltered pixels. To fix this an improved methodology is proposed to increase the performance of the change detection process based on image fusion by using the ADWT with FCM clustering. Firstly, images are picked up at the geographical area at various timings. Consequently, difference images are generated by performing ratio (Log-Ratio and Mean-Ratio) operations. The image fusion is performed with two difference images, i.e., one is the result of the Log-Ratio operator and the other one is the result of MeanRatio operator. This image fusion is performed by choosing the optimized filter coefficients rather than conventional wavelet filter coefficients (Daubechies, Haar). The process of performing DWT by choosing the optimized filter coefficients is named as Adaptive Discrete Wavelet Transform (ADWT). After image fusion, the early image is be reconstructed by using the IDWT. Clustering is be performed for the reconstructed image and the resulting image is compared with the ground truth image. Finally, the performance is measured in terms of measuring parameters.

\section{Literature review and motivation of the problem}

In 2019, $\mathrm{Z}$ h a o, Li ing and Li [22] have implemented a novel unsupervised Change Detection (CD) technique of flood mapping in SAR images. In 2018, Li et al. [23] have developed an unsupervised SAR change detection framework for spotting out the changes in SAR with the aid of robust semi-non-negative matrix factorization with TV. In 2017, Lazarov and Minchev [24] proposed ISAR Image Recognition Algorithm and Neural Network Implementation. In 2014, Hou et al. [25] have invented a novel unsupervised SAR change detection approach on the based on GLR-IM and compressed projection for transformation discovery in SAR images. In 2014, Zheng et al. [26] have introduced a simple and effective unsupervised approach for CD in SAR images using the KMC. In 2013, Z h a n g, $\mathrm{Ch}$ e $\mathrm{n}$ and Me $\mathrm{ng}$ [27] have developed a robust as well as a fast-unsupervised SAR change-detection framework for detecting the modifications in SAR images. In 2012, Gong, Z hou and Ma [28] have formulated an unsupervised CD in SAR images based on distribution-free approach for determining the modifications in SAR images using the image fusion strategy as well as a novel fuzzy clustering algorithm. In 2009, Moser and Serpico [29] have formulated a new model for multichannel amplitude SAR image contextual change-detection in an unsupervised and automatic manner.

The main objective of this proposal is to improve the overall performance of CD by maximizing the accuracy through the proposed methodology over conventional methodologies. Change detection process has been performed for Ottawa data set captured from the geographical area of Canada. Two multitemporal SAR images have been taken to detect the changes, and changes occurred due to floods, land cover 
changes. Hence, this process could be used for society to identify the damages, which would occur due to natural disasters or changes in the earth's surface.

\section{Proposed methodology}

The proposed methodology is to improve the overall performance of the change detection process by using image fusion based on ADWT and segmentation by using Fuzzy c-means clustering. ADWT is performed by choosing optimized filter coefficients of wavelets based on the Genetic Algorithm (GA), Particle Swarm Optimization Algorithm (PSO Algorithm) and AntLion Optimization Algorithm (ALO Algorithm).

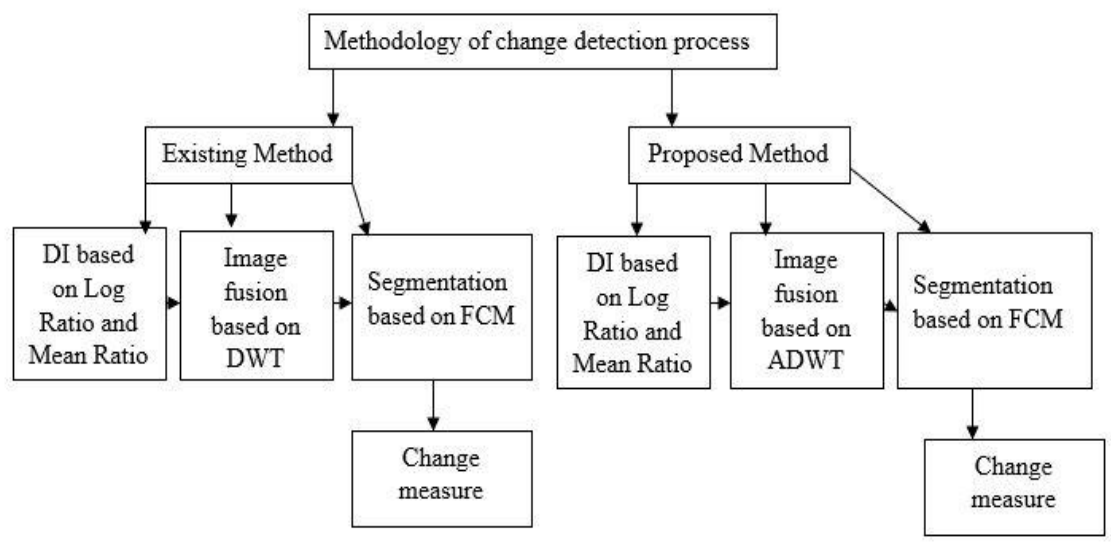

Fig. 1. Proposed methodology

The proposed methodology is illustrated in Fig. 1 and the following steps are involved in this process:

Step 1. Difference images are generated through the Log-Ratio operator and Mean-Ratio operator.

Step 2. Image fusion using conventional DWT and ADWT (using GA, PSO, ALO).

Step 3. Segmentation using FCM clustering.

Step 4. Measurement comparison of ground truth image and segmented image.

As illustrated in Fig. 2. Optimized filter coefficients have been chosen based on GA, PSO and Antlion optimization algorithms. The decisive thing is the selection of filter coefficients based on satisfying the orthogonality property to reconstruct the original image by minimizing the error. As it is known some content of input will be lost during the process of DWT which causes unfaithful reconstruction of the original image. In this paper, it is proposed that the loss in the DWT process could be reduced through the optimal selection of filter coefficients. After image fusion process, segmentation has been done based on Fuzzy C-Means clustering (FCM). In the latter, 
the segmented image is compared with the ground truth image, and performance has been analysed [2-5].

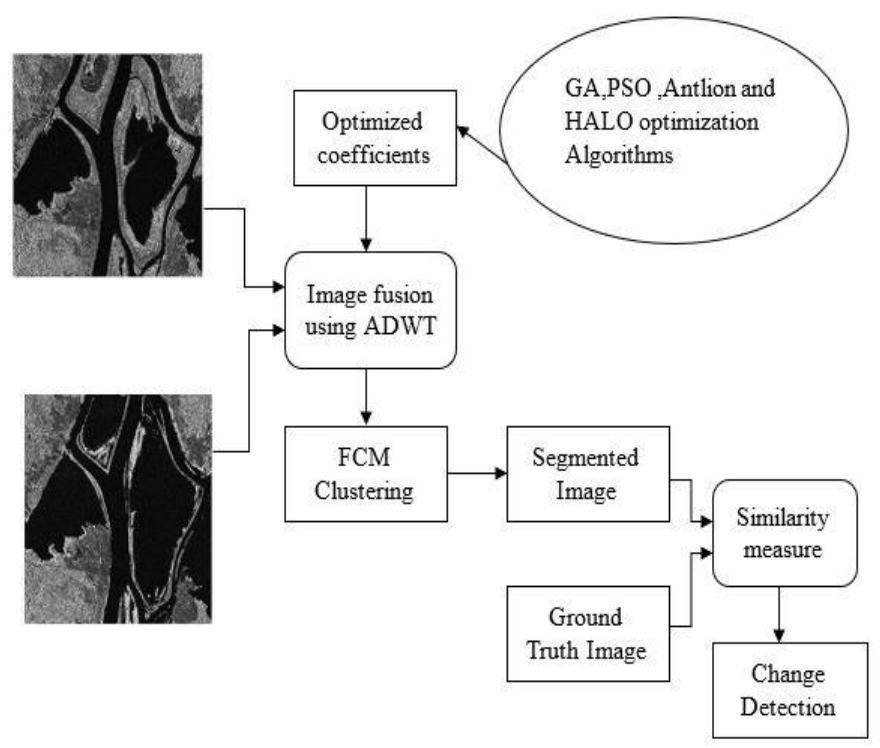

Fig. 2. Overall Architecture of the proposed methodology

\subsection{Adaptive Discrete Wavelet Transform (ADWT)}

The proposed ADWT [2-5] is performed by adapting optimized filter coefficients through the optimization algorithms rather than using conventional wavelet filter coefficients (Daubechies, Haar). As depicted in Fig. 3. The hidden thing in choosing ADWT is to reduce the quantization error during the DWT process, proportionally minimizing error in the reconstructed image to achieve faithful reconstruction.

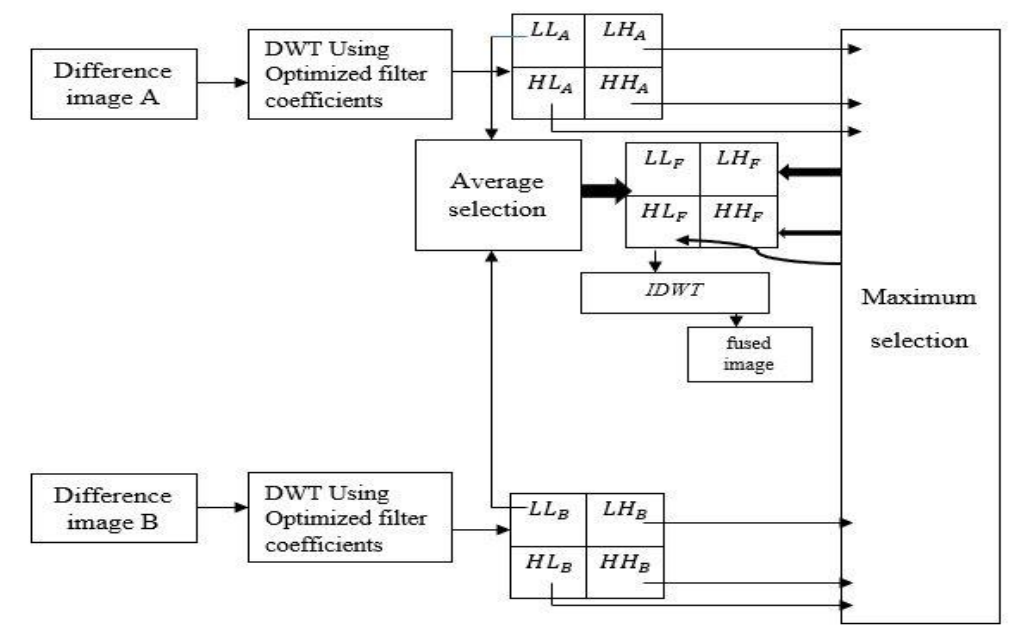

Fig. 3. Proposed 2D-ADWT architecture 
The DWT is used to redistribute the energy of a given signal. The major components of DWT are given as (1) scaling function $\phi(t)$ and (2) wavelet function $\psi(t)$. Instead of using conventional wavelet coefficients (Hi_D, Li_D) in image fusion, optimized filter coefficients $\left\{\mathrm{Hi} \_\mathrm{D}(\mathrm{OP}), \mathrm{Li} \_\mathrm{D}(\mathrm{OP})\right\}$ are optimally chosen through GA, PSO and ALO Algorithms. ADWT-based image fusion is executed for difference Image (DI) A (Log-ratio) and difference image B (Mean-Ratio) and the Inverse Discrete Wavelet Transform (IDWT) is be performed, to reconstruct the original image by using images $\mathrm{LL}_{\mathrm{F}}, \mathrm{LH}_{\mathrm{F}}, \mathrm{HL}_{\mathrm{F}}$, and $\mathrm{HH}_{\mathrm{F}}$. The decomposition images after performing 2D-ADWT are abbreviated as follows:

- $L L_{F}$ is an approximation image by taking the average of $L_{A}$ and $L L_{B}$ images;

- $\mathrm{LH}_{\mathrm{F}}$ is Horizontal image by selecting the maximum of $\mathrm{LH}_{\mathrm{A}}$ and $\mathrm{LH}_{\mathrm{B}}$ images;

- $\mathrm{HL}_{\mathrm{F}}$ is a vertical image by selecting the maximum of $\mathrm{HL}_{\mathrm{A}}$ and $\mathrm{HL}_{\mathrm{B}}$ images;

- $\mathrm{HH}_{\mathrm{F}}$ is Diagonal image by selecting the maximum of $\mathrm{HH}_{\mathrm{A}}$ and $\mathrm{HH}_{\mathrm{B}}$ images;

- $\mathrm{LL}_{\mathrm{A}}$ and $\mathrm{LL}_{\mathrm{B}}$ are the decomposition images of approximation images of DI of $\mathrm{A}$ and $\mathrm{DI}$ of $\mathrm{B}$, respectively;

- $\mathrm{LH}_{\mathrm{A}}$ and $\mathrm{LH}_{\mathrm{B}}$ are the decomposition images of horizontal images of DI of $\mathrm{A}$ and $\mathrm{DI}$ of $\mathrm{B}$, respectively;

- $\mathrm{HL}_{\mathrm{A}}$ and $\mathrm{HL}_{\mathrm{B}}$ are the decomposition images of vertical images of DI of $\mathrm{A}$ and $\mathrm{DI}$ of $\mathrm{B}$, respectively;

- $\mathrm{HH}_{\mathrm{A}}$ and $\mathrm{HH}_{\mathrm{B}}$ are the decomposition images of Diagonal images of DI of A and $\mathrm{DI}$ of $\mathrm{B}$, respectively.

The overall performance analysis is measured between conventional DWT (using Daubechies wavelets) and ADWT (using optimized filter coefficients through GA, PSO and ALO Algorithms). In [7-10] Daubechies wavelet with two vanishing moments is called as Daubechies 2 wavelet. The transfer functions of decomposition filters are expressed in the next equations:

$$
\begin{aligned}
& H(z)=h_{-2} z^{2}+h_{-1} z^{1}+h_{0}+h_{1} z^{-1}, \\
& G(z)=-h_{1} z^{2}+h_{0} z-h_{-1}+h_{-2} z^{-1} .
\end{aligned}
$$

The transfer function of reconstruction filter coefficients is expressed in the equations

$$
\begin{gathered}
\bar{H}(z)=h_{1} z+h_{0}+h_{-1} z^{-1}+h_{-2} z^{-1}, \\
\bar{G}(z)=-h_{1} z^{-2}+h_{0} z^{-1}-h_{-1}+h_{-2} z^{1},
\end{gathered}
$$

where

where $\bar{H}(z)=H\left(z^{-1}\right)$ and $\bar{G}(z)=G\left(z^{-1}\right)$.

$$
\begin{gathered}
h_{0}=\frac{3-\sqrt{3}}{4 \sqrt{2}}, \\
h_{1}=\frac{1-\sqrt{3}}{4 \sqrt{2}}, \\
h_{-1}=\frac{3+\sqrt{3}}{4 \sqrt{2}}, \\
h_{-2}=\frac{1+\sqrt{3}}{4 \sqrt{2}},
\end{gathered}
$$

Instead of using standard wavelet filter coefficients (Equations (5)-(8)) optimized filter coefficients could be used by satisfying the orthogonality property. 


\subsection{Optimization algorithms for performing ADWT}

Three types of optimization algorithms have been adapted for performing ADWT. The overall parameters of optimization are illustrated in Table 1.

Table 1. Function details for the Optimization process

\begin{tabular}{|l|l|r|}
\hline \multicolumn{2}{|l|}{ Testing function: SPHERE } \\
\hline No & Parameter & Value \\
\hline 1 & Search Agents & 30 \\
\hline 2 & Max iteration & 100 \\
\hline 3 & Lower bound & -10 \\
\hline 4 & Upper bound & 10 \\
\hline 5 & Dimension & 10 \\
\hline
\end{tabular}

\subsubsection{Conventional Antlion Algorithm (ALO)}

This algorithm has been developed based on the searching mechanism for the prey of antlions $[18,19,21]$. ALO is a new stochastic algorithm that has been developed from the inspiration of the antlions hunting mechanism. In this regard, antlions and ants act as searching agents for hunting the prey. The steps, which are involved in hunting prey, are given as random walk of the ant, forming paths of traps, enmeshment of ants in traps, capturing the prey and rebuilding the trap. The mathematical model of the algorithm is elaborated as follows.

In the process of hunting ant moves by a random walk and the optimization process is expressed as

$$
A_{i}=\left[0 ; s(1) ; s(1)+s(2) ; \ldots \sum_{j=1}^{T-1} s(j) ; \sum_{j=1}^{T} s(j)\right],
$$

where $i=1, \ldots$, Dim, Dim is the dimension of ant and antlions, $T$ is the maximum iteration;

$$
\begin{gathered}
A_{i}=\left[A_{1} \ldots A_{\text {dim }}\right], \\
A_{i}=(T+1) \times 1 \text { matrix },
\end{gathered}
$$

where $s(j)$ is expressed as

$$
s(j)=\left\{\begin{array}{r}
1 \text { if rand }>0.5, \\
-1 \text { if rand } \leq 0.5,
\end{array}\right.
$$

where "rand" indicates random numbers in the interval $[0,1]$. Based on Lower Bound (LB) value and Upper Bound (UB) value the random walk of search agents will be converted into a possible search space, which is calculated as per Equation (12). The Bound of $i$-th ant is

$$
B_{i}=\frac{A_{i}-x_{i}}{z_{i}-x_{i}} \times\left(d_{i}-c_{i}\right)+c_{i},
$$

$A_{i} \in[0,1]$. By changing the ant movement antlions trap the ant. This can be formulated as

$$
\begin{aligned}
& c=c^{\prime}+\text { Antlion, } \\
& d=d^{\prime}+\text { Antlion, }
\end{aligned}
$$

where $c^{\prime}$ and $d^{\prime}$ are lower limit and upper limit of the movement of the ant, respectively. Based on the Roulette wheel the position of the antlion will be selected 
[19]. Once ants are trapped then the radius of ant movement would be decreased and formulated as Equations (14)-(15);

$$
\begin{aligned}
& c^{\prime}=\frac{\mathrm{LB}}{10^{w} \times(t / T)^{\prime}}, \\
& d^{\prime}=\frac{\mathrm{UB}}{10^{w} \times(t / T)},
\end{aligned}
$$

where $w$ is constant value and $t \in[0,1]$.

Elitism is adopted in this algorithm, therefore based on the Roulette wheel and Elitism ant updates its new position. Hence, the new position of the ant could be represented as

$$
\text { Ant }=\frac{R_{A}+R_{B}}{2},
$$

where $R_{A}, R_{B}$ are the random walk of antlion based on the Roulette wheel and Elitism, respectively.

\subsubsection{Hybridized Antlion Algorithm (HALO Algorithm)}

In this paper, the proposed algorithm is named as HALO (Hybridized ALO) Algorithm which is implemented to perform ADWT through selection of optimized coefficients. The concept in developing this algorithm is hybridizing the Artificial Bee Colony algorithm with ALO Algorithm. In conventional ALO the new position of the ant will be updated as per Equation (18) but to reduce the search space the new position will be updated according to HALO Algorithm is according to next equation: (19) $A_{i, j}=\frac{R_{A}+R_{B}}{2}+\operatorname{rand}(0,1)\left[\left(R_{A \max }, j-R_{A \min }, j\right)-\left(R_{E \max }, j-R_{E \min }, j\right)\right]$.

After calculating the fitness value according to $\mathrm{ABC}$ algorithm $R_{A \max }$ and $R_{E \max }$ are current positions of the ant based on $\mathrm{ABC}$ Algorithm respectively and $R_{A \min }, R_{E \min }$ are previous positions of ant according to conventional ALO. In this way the performance of ALO Algorithm has been improved.

The execution of HALO Algorithm is explained as in Fig. 4.

Step 1. Initialize population size $A_{i}=\left[A_{1} \ldots \ldots \ldots A_{\operatorname{dim}}\right]$

Step 2. Calculate fitness of both ant and antlions

Step 3. Based on roulette wheel select antlion for every ant

Step 4. Update the position based on Equation (18)

Step 5. Calculate the fitness of all ants

Step 6. If the end criterion is satisfied, return the elite. Otherwise, return to Step 3

Fig. 4. Pseudocode of HALO Algorithm

\subsubsection{Fuzzy c-Means clustering (FCM)}

In this paper, the FCM clustering technique has been adapted for segmentation. It is a known fact that changed pixels and unchanged pixels could be separated by segmentation. The composing of FCM clustering is illustrated in Fig. 4. In order to reduce the search space. 
Step 1. Initialize $=\left[t_{i j}\right]$ matrix $T^{0}$

Step 2. At $k$-step calculate the centre vectors $B^{(K)}=\left[B_{i}\right]$ with $T^{(k)}$,

Step 3. Update $T^{(k)}, T^{(k-1)}$,

$$
B_{j}=\frac{\sum_{i=1}^{N} t_{i j}{ }^{m} \cdot x_{i}}{\sum_{i=1}^{N} t_{i j}{ }^{m}}
$$

$$
t_{i j}=\frac{1}{\sum_{k=1}^{b}\left(\frac{\left\|x_{i}-b_{j}\right\|}{\left\|x_{i}-b_{j}\right\|}\right)^{\frac{2}{m-1}}}
$$

Step 4. if $\left\|T^{(k+1)}-T^{k}\right\|<\varepsilon$ then stop; otherwise return to Step 2

Fig. 5. Composing of FCM clustering

\section{Results and discussions}

In this paper to endorse the performance of the proposed methodology over the conventional method (image fusion with Daubechies wavelets) satellite images with SAR, sensors have been taken into account. Ottawa data set is taken as data set to validate the proposed methodology. Fig. 6a shows the geographical image of Canada and Fig. 6 b shows its capital city Ottawa. Fig. 7 shows the Radar sat images of Ottawa city in Canada. Fig. 7a shows the satellite image before occurrence of the flood, the image after the flood is shown in a Fig. 7b, and the majority of the affected areas are marked in red. The corresponding ground truth image is shown in a Fig. 7c.

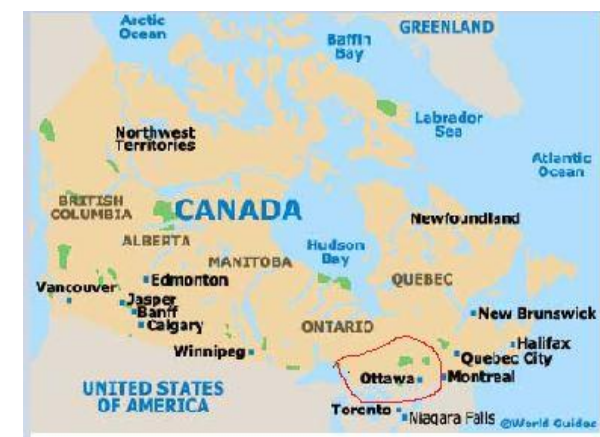

(a)

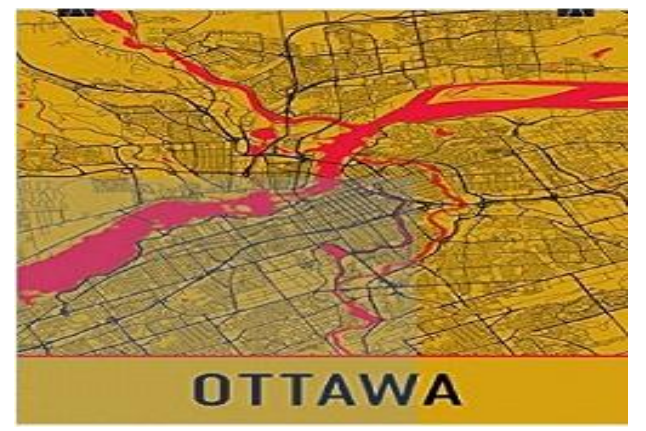

(b)

Fig. 6. Geographical map of Canada (a); Ottawa city map (b)

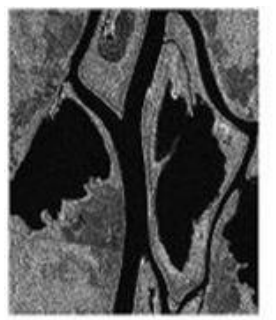

(a) Before flood

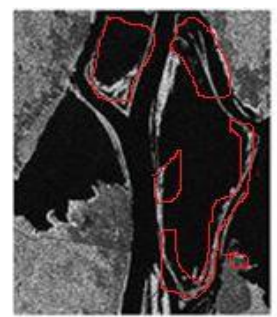

(b) After flood

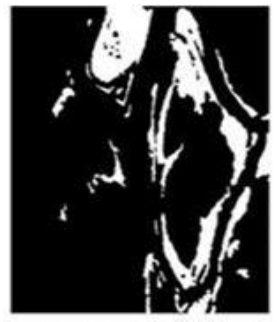

(c) Ground truth image

Fig. 7. Ottawa data set 
As illustrated in Fig 1 the initial step in the proposed methodology is generating the DI by Log-ratio operator and Mean-ratio operator. The difference images are depicted in Fig. 7. Log-ratio operator DI is shown in Fig. 8 a and the Mean-ratio operator DI is illustrated in a Fig. 8b.

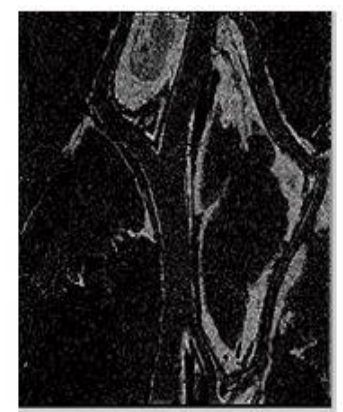

(a)

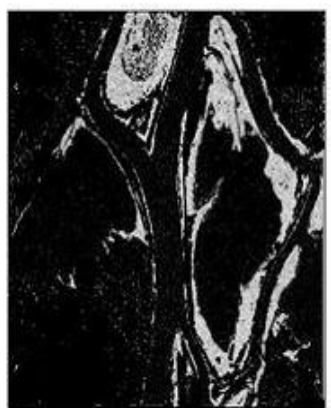

(b)

Fig. 8. Difference Images: Log-ratio operator (a); Mean-ratio operator (b)

As per Step 2, image fusion will be performed based on conventional DWT (with Daubechies 2 wavelets) and ADWT and the corresponding images are illustrated in Fig. 9.

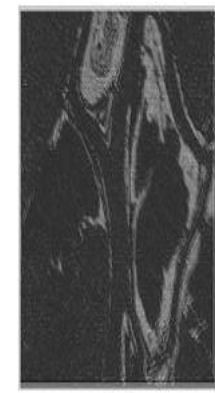

(a)

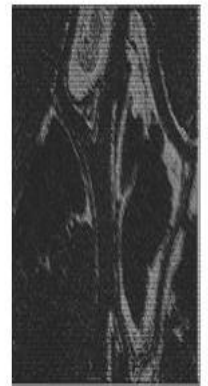

(b)

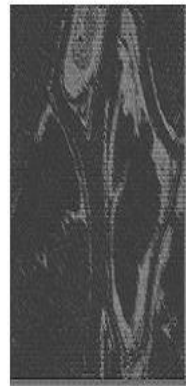

(c)

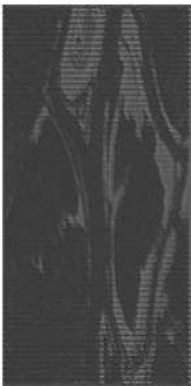

(d)

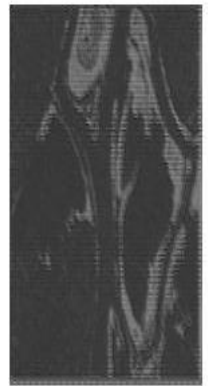

(e)

Fig. 9. DWT (a); ADWT_GA (b); ADWT_PSO (c); ADWT_ALO (d); ADWT_HALO (e)

Image segmentation has been employed to differentiate changed pixels and unchanged pixels. In this regard, FCM clustering has been performed for segmentation and the resulting output images are exploited in a Fig. 10.

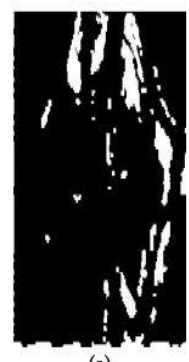

(a)

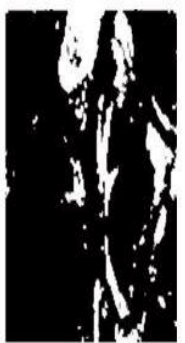

(b)

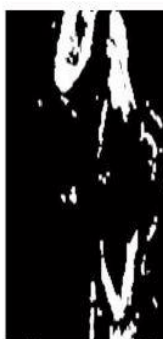

(c)

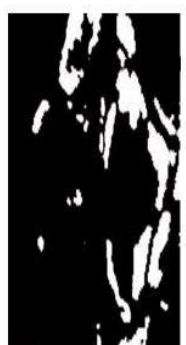

(d)

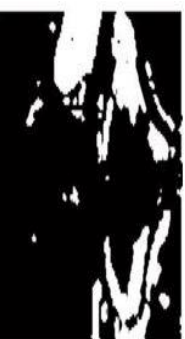

(e)

Fig. 10. DWT_FCM (a); GA_FCM (b); PSO_FCM (c); ALO_FCM (d); HALO_FCM (e) 


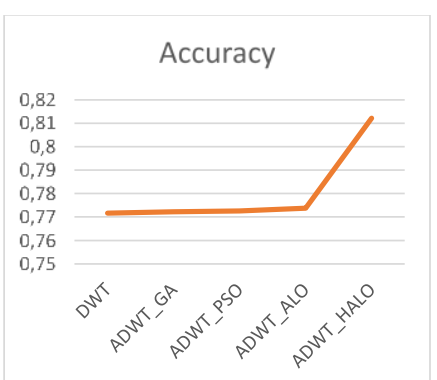

(a)

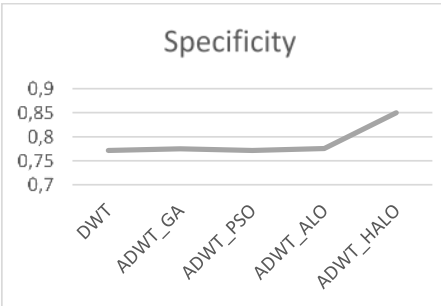

(c)

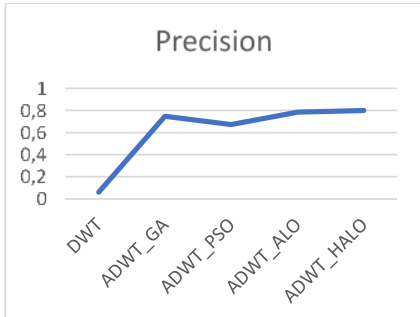

(e)

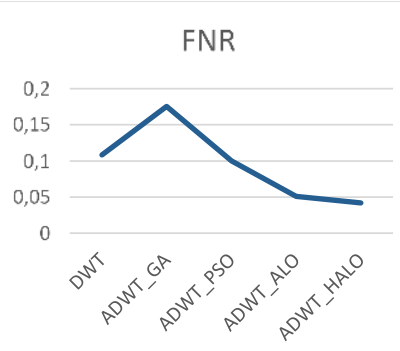

(g)

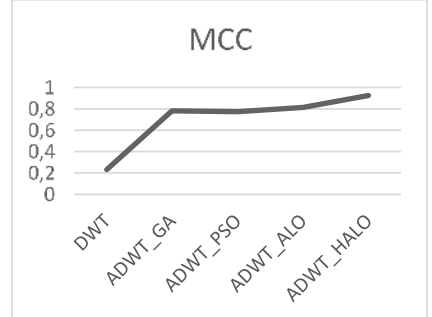

(i)

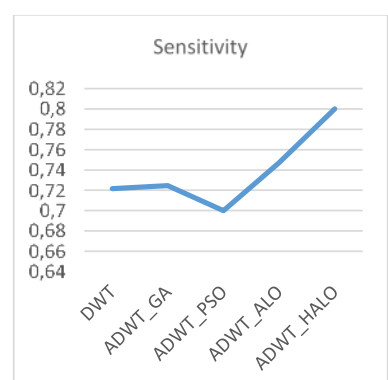

(b)

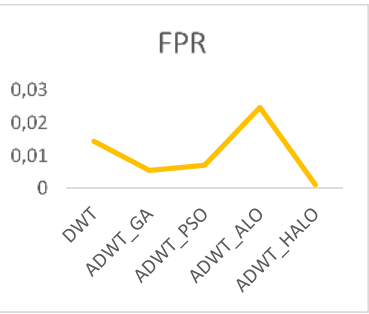

(d)

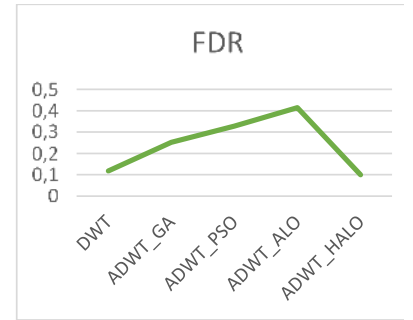

(f)

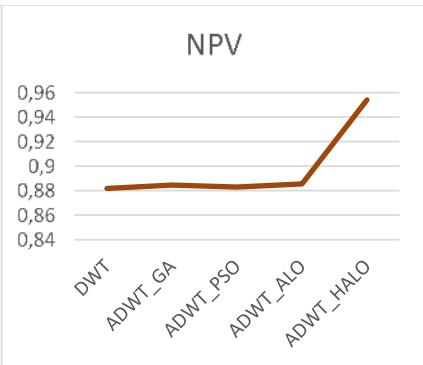

(h)

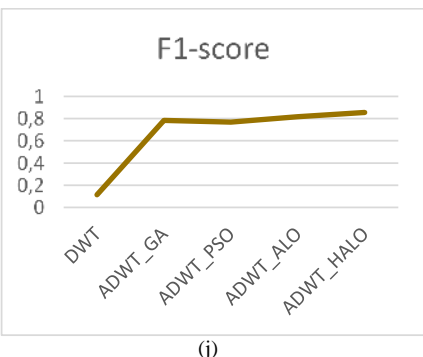

(j)

Fig. 11. Graphical representation of proposed methodology over the conventional method 
Table. 2. Performance analysis of proposed methodology over conventional DWT

\begin{tabular}{|c|c|c|c|c|c|}
\hline Measure & DWT & ADWT_GA & ADWT_PSO & ADWT_ALO & ADWT_HALO \\
\hline Sensitivity & 0.7217 & 0.7245 & 0.7 & 0.7471 & 0.8 \\
\hline Accuracy & 0.7716 & 0.7722 & 0.7726 & 0.7737 & 0.8122 \\
\hline Specificity & 0.7714 & 0.7746 & 0.7715 & 0.7754 & 0.8501 \\
\hline FPR & 0.0143 & 0.0054 & 0.007 & 0.0246 & 0.001 \\
\hline Precision & 0.0607 & 0.7475 & 0.6723 & 0.786 & 0.7991 \\
\hline FDR & 0.1174 & 0.2525 & 0.3277 & 0.414 & 0.1 \\
\hline FNR & 0.1081 & 0.1755 & 0.1 & 0.0508 & 0.0421 \\
\hline NPV & 0.8817 & 0.8846 & 0.883 & 0.8854 & 0.9541 \\
\hline MCC & 0.2308 & 0.7807 & 0.7738 & 0.8138 & 0.9243 \\
\hline F1-score & 0.114 & 0.7841 & 0.7687 & 0.8164 & 0.8556 \\
\hline
\end{tabular}

\section{Conclusion}

As in the previous literature, the majority of researchers opt two types of ratio operators (Log-Ratio operator and Mean-Ratio operator) to generate DI for CD in SAR images. However, Log ratio operator does not represent altered pixels and unaltered pixels symmetrically, in the same way, the Mean-ratio operator does not represent difference images smoothly. To fix these problems image fusion has been introduced. Even though the difference image is fused but some of the original image information will be lost in the DWT process. By adapting the ADWT technique, maximum error could be reduced in the DWT process. Finally, it has been proved that ADWT (using GA, PSO, ALO and HALO optimization Algorithms) can give more accuracy than a conventional DWT.

\section{References}

1. Gon g, M., Z. Zhou, J. Ma. Change Detection in Synthetic Aperture Radar Images Basedon Image Fusion and Fuzzy Clustering. - IEEE Transactions on Image Processing, Vol. 21, 2012, No 4, pp. 2141-2151.

2. Jakka, T. K., Y. Mallikarjuna Reddy, B. Prabhakara Rao. GWDWT-FCM: Change Detection in SAR Images Using Adaptive Discrete Wavelet Transform with Fuzzy c-Mean Clustering. - Journal of the Indian Society of Remote Sensing, Vol. 47, 2019, No 3, pp. 379-390.

3. K u m a r, J. T., Y. M. R e d d y, B. P. R a o. Image Fusion of Remote Sensing Images Using ADWT with ABC Optimization Algorithm. - International Journal of Innovative Technology and Exploring Engineering (IJITEE), Vol. 8, 2019, Issue 11. ISSN: 2278-3075.

4. K u m a r, J. T., Y. M. R e d d y, B. P. R a o. Change Detection in Sarimages Based on Artificial Bee Colony Optimization with Fuzzy c-Means Clustering. - International Journal of Recent Technology and Engineering (IJRTE), Vol. 7, 2018, Issue 4. ISSN: 2277-3878.

5. K u mar, J. T., Y. M allikarjun a Redd y, B. Prabhakara Rao. WHDA-FCM: Wolf Hunting-Based Dragonfly with Fuzzy c-Mean Clustering for Change Detection in SAR Images. - The Computer Journal, Section B: Computer and Communications Networks and Systems, Vol. 63, February 2020, Issue 2, pp. 308-321.

6. Ingla d a, J., G. Merci e r. A New Statistical Similarity Measure for Change Detection in Multitemporal SAR Images and Its Extension to Multiscale Change Analysis. - IEEE Trans. Geosci. Remote Sens., Vol. 45, 2017, No 5, pp. 1432-1445. 
7. S ing h, A. Digital Change Detection Techniques Using Remotely Sensed Data. - International Remote Sensing, Vol. 10, 1989, No 6, pp. 989-1003.

8. Rig n o t, E. J. M., J. J. V a n Z y l. Change Detection Techniques for ERS-1 SAR Data. - IEEE Trans. Geosci. Remote Sens., Vol. 31, 1993, No 4, pp. 896-906.

9. Mirjalili 1, S. Dragonfly Algorithm: A New Meta-Heuristic Optimization Technique for Solving Single-Objective, Discrete, and Multi-Objective Problems. - Neural Comput\&Applic, Vol. 27, 2016, pp. 1053-1073.

10. SreeRanjini, K. S., S. Murugan. Memory-Based Hybrid Dragonfly Algorithm for Numerical Optimization Problems. - Expert Systems with Applications, Vol. 83, 2017, pp. 63-78.

11. Ç i g d e m i n a n, A., H. G u 1 k a n. A Modified Dragonfly Optimization Algorithm for Single- and Multiobjective Problems Using Brownian Motion. - Hindawi Computational Intelligence and Neuroscience, 2019. Article ID 6871298. 17 p.

12. V r i o n i s, T. D., X. I. K o u t i v a, N. A. V o v o s. A Genetic Algorithm-Based Low Voltage RideThrough Control Strategy for Grid Connected Doubly Fed Induction Wind Generators. - IEEE Transactions on Power Systems, Vol. 29, 2014, No 3, pp. 1325-1334.

13. Hu i, Z., Y. Fe i. A Novel Fuzzy Clustering Recommendation Algorithm Based on PSO. Cybernetics and Information Technologies, Vol. 14, 2014, No 1, pp. 108-117.

14. Z h a n g, J., P. X i a. An Optimized Scheduling Algorithm on a Cloud Workflow Using a Discrete Particle Swarm. -Cybernetics and Information Technologies, Vol. 14, 2014, No 1, pp. 25-39.

15. Y a n, W., S. S h i, L. P a n, G. Z h a n g. Unsupervised Change Detection in SAR Images Based on Frequency Difference and a Modified Fuzzy c-Means Clustering. - International Journal of Remote Sensing, Vol. 39, 2018, No 10, pp. 3055-3075.

16. Q i u, F., J. B e r g l u n d, J. R. J e n s e n, P. T h a k k a r, D. R e n. Speckle Noise Reduction in SAR Imagery Using a Local Adaptive Median Filter. -GIScience and Remote Sensing, Vol. 3, 2004, pp. 244-266.

17. Z h u a n g, H., Z. T a n, K. D e n g, H. F a n. It is a Misunderstanding that Log-Ratio Outperforms Ratio in Change Detection of SAR Images. - European Journal of Remote Sensing, Vol. 52, 2019, No 1, pp. 484-492.

18. Vijaya Geetha, R., S. Kalaivani. Laplacian Pyramid-Based Change Detection in Multitemporal SAR Images. - European Journal of Remote Sensing, Vol. 5, 2019.

19. L i u, T., C. Q. G u o, Y. Y u a n, W. L i, Q. Y a n. An Improved Ant Lion Optimization Algorithm and Its Application in Hydraulic Turbine Governing System Parameter Identification. Energies, Vol. 11, 2018, pp. 1-15.

20. Vrionis, T., X. Koutiva, Nicholas. A Genetic Algorithm-Based Low Voltage RideThrough Control Strategy for Grid Connected Doubly Fed Induction Wind Generators. - IEEE Transactions on Power Systems, 2014, Vol. 29, No 3, pp. 1325-1334.

21. M i r j a li li, S. The Ant Lion Optimizer. - Adv. Eng. Software, Vol. 83, 2015, pp. 80-98.

22. Z h a o, M., Q. L i n g, F. L i. An Iterative Feedback-Based Change Detection Algorithm for Flood Mapping in SAR Images. - IEEE Geoscience and Remote Sensing Letters, Vol. 16, February 2019, No 2, pp. 231-235.

23. Li, H., Q. Z h a o, G. Y a n g, K. F u, W. J. E m e r y. Robust Semi-NMF with Total Variation for Unsupervised SAR Image Change Detection. - Electronics Letters, Vol. 54, 12.07.2018, No 14, pp. 892-894.

24. Lazarov, A., C. Minchev. ISAR Image Recognition Algorithm and Neural Network Implementation. - Cybernetics and Information Technologies, Vol. 17, 2017, No 4, pp. 183-199.

25. H o u, B., Q. W e i, Y. Z h e n g, S. W a n g. Unsupervised Change Detection in SAR Image Based on Gauss-Log Ratio Image Fusion and Compressed Projection. - IEEE Journal of Selected Topics in Applied Earth Observations and Remote Sensing, Vol. 7, August 2014, No 8, pp. 3297-3317.

26. Z h e n g, Y., X. Z h a n g, B. Ho u, G. Li u. Using Combined Difference Image and \$k\$-Means Clustering for SAR Image Change Detection. - IEEE Geoscience and Remote Sensing Letters, Vol. 11, March 2014, No 3, pp. 691-695. 
27. Z h a n g, X., J. Ch e n, H. M e n g. A Novel SAR Image Change Detection Based on Graph-Cut and Generalized Gaussian Model. - IEEE Geoscience and Remote Sensing Letters, Vol. 10, January 2013, No 1, pp. 14-18.

28. Gong, M., Z. Zho u, J. M a. Change Detection in Synthetic Aperture Radar Images Based on Image Fusion and Fuzzy Clustering. - IEEE Transactions on Image Processing, Vol. 21, April 2012, No 4, pp. 2141-2151.

29. M o s e r, G., S. B. S e r p i c o. Unsupervised Change Detection From Multichannel SAR Data by Markovian Data Fusion. - IEEE Transactions on Geoscience and Remote Sensing, Vol. 47, July 2009, No 7, pp. 2114-2128.

Received: 20.07.2020; Second Version: 26.01.2021; Accepted: 23.02.2021 